\title{
КЛИНИЧЕСКИЕ ХАРАКТЕРИСТИКИ И ЭФФЕКТИВНОСТЬ ЛЕЧЕНИЯ АКРОМЕГАЛИИ У ПАЦИЕНТОВ В УДМУРТСКОЙ РЕСПУБЛИКЕ
}

\author{
Нуруллина Г.М., Ахмадуллина Г.И., Николаева А.Р.
}

ФГБОУ ВО «Ижевская государственная медицинская академия» Минздрава России, Ижевск

ЦЕЛь: изучить клинические характеристики и эффективность лечения у пациентов с акромегалией в Удмуртской Республике.

МАТЕРИАЛЫ И МЕТОДЫ: проанализированы данные стационарного и амбулаторного обследования с 2020 по 2021 г. 39 человек с акромегалией, среди которых 29 женщин (74,36\%), 10 мужчин (25,64\%),

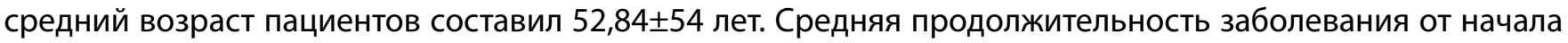

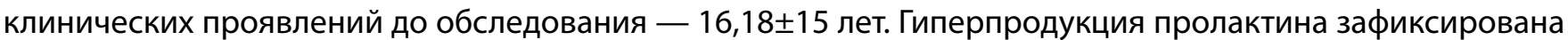
у 31,57\% пациентов, АКТГ - у 2,63\% при выявлении акромегалии по данным медицинской документации.

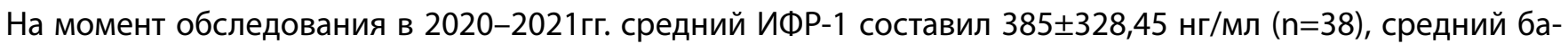

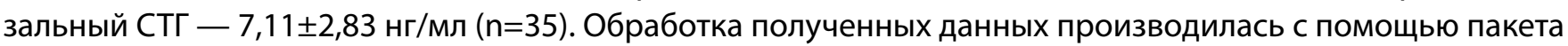
программ STATISTICA 10,0 фирмы StatSoft.

PЕзУЛЬТАТЫ: у 81,08\% пациентов наблюдалась артериальная гипертензия, у 21,62\% - акромегалическая кардиомиопатия, 10,81\% пациентов перенесли инсульт, 2,7\% - инфаркт миокарда. Частота сахарного диабета составила 37,83\%, нарушения толерантности к углеводам - 10,81\%. Диффузный зоб диагностирован у 51,35\% пациентов, узловой зоб - у 72,97\%. Артралгии наблюдались у 70,27\% пациентов, патологические переломы - у 21,62\%, храп - у 38,88\%, головные боли - у 86,11\%, бесплодие и нарушение менструального цикла у 21,62\%, синдром запястного канала - у 11,11\%, зрительные нарушения - у 40,54\%. Пангипопитуитаризм обнаружен у 24,32\% пациентов, вторичный гипотиреоз - у 24,32\%, вторичный гипокортицизм - у 21,62\%, вторичный гипогонадизм - у 40,54\%, центральный несахарный диабет - у 2,7\%, дефицит пролактина - у 8,3\%, дефицит гормона роста - у 2,56\%.

За период заболевания нейрохирургическое лечение было проведено у 66,66\% (26 чел.), повторное нейрохирургическое вмешательство - у 4 человек из 26 (10,25\%), лучевая терапия была у 9 чел. (23,07\%). Медикаментозную терапию соматостатином пролонгированного действия получают 22 человека (56,41\%), среди них 1 человек вводит препарат в дозировке 10 мг в 28 дней (4,54\%), 15 человек в дозировке 20 мг/28 дней (68,18\%), 1 человек - в дозировке 30 мг/28 дней (4,54\%), 5 человек - в дозировке 40 мг/28 дней (22,72\%). 1 из 39 пациентов получает ланреотид в дозировке 120 мг/28 дней (2,56\%), 7 человек - терапию каберголином (17,94\%). У большинства больных отсутствует ремиссия акромегалии (у 57,89\%), у 2,63\% наблюдается неполная ремиссия, ремиссия достигнута у 39,47\%.

ВЫВОДЫ: наиболее часто выявлялись следующие клинические проявления акромегалии - артериальная гипертензия, артралгия, цефалгия, нарушения углеводного обмена, тиреопатии, что является типичным для данного заболевания. У большинства пациентов не достигнута ремиссия акромегалии, что, возможно, связано с отсутствием у 33,34\% нейрохирургического лечения, а также с клинической инертностью. 